\title{
El papel de la motivación en la predicción del conocimiento procedimental en jugadores de baloncesto*
}

\section{The Role of the Motivation in the Prediction of Procedimental Knowledge in Basketball Players}

Recepción: 12 Enero 2012 | Aprobación: 18 Junio 2013

\author{
Eduardo Cervelló Gimeno** \\ Universidad Miguel Hernández de Elche, España \\ David González-Cutre \\ Universidad Miguel Hernández de Elche, España \\ Juan Antonio Moreno Murcia \\ Universidad Miguel Hernández de Elche, España \\ Damián Iglesias Gallego \\ Universidad de Extremadura, España
}

\footnotetext{
**Universidad Miguel Hernández de Elche. Centro de Investigación del Deporte. Avenida de la Universidad, s. n. 03202 Elche (Alicante). Tel: +34 96655 8878. Correo electrónico: ecervello@umh.es
}

\section{RESUMEN}

El objetivo de este estudio fue analizar el poder de predicción de las creencias implícitas de habilidad, las orientaciones de meta y la percepción de competencia deportiva sobre el conocimiento procedimental en jugadores de baloncesto. En la investigación participaron 339 jugadores de edades comprendidas entre los 12 y 15 años. Se les midió la orientación motivacional (POSQ), las creencias de habilidad en el deporte (CNAAQ-2), la competencia deportiva y el conocimiento procedimental en baloncesto (CCPB). Los resultados mostraron que la creencia incremental de habilidad predecía positivamente el conocimiento procedimental, mientras que la creencia de entidad lo hacía de forma negativa. La relación entre las creencias de habilidad y el conocimiento procedimental fue directa, sin mediación de las orientaciones de meta y la competencia percibida. Los resultados se discuten en relación con el papel de la motivación sobre el conocimiento procedimental en el deporte.

Palabras clave

creencias de habilidad, competencia, motivación, deporte, baloncesto, conocimiento procedimental.

\begin{abstract}
The objective of this study was to analyze the prediction of ability beliefs, goal orientations and perceptions of athletic competence on procedural knowledge in basketball players. The research involved 339 players aged between 12 and 15. The motivational orientation (POSQ), the ability beliefs in sport (CNAAQ-2), sport competence and procedural knowledge in basketball (CCP) were measured. The results of the structural equation modeling, showed that incremental ability belief predicted positively procedural knowledge, while the entity ability belief predicted procedural knowledge negatively. The relationship between the ability beliefs and procedural knowledge was direct, without the mediation of goal orientations and perceived competence. The results are
\end{abstract}


Eduardo Cervelló Gimeno, David González-Cutre, Juan Antonio Moreno Murcia, Damián Iglesias Gallego.

discussed in relation to the role of motivation on procedural knowledge on the sport.

Keywords

ability beliefs, competence, motivation, sports, basketball, procedural knowledge.

Para citar este artículo: Cervelló Gimeno, E., González-Cutre, D., Moreno Murcia, J. A., \& Iglesias Gallego, D. (2016). El papel de la motivación en la predicción del conocimiento procedimental en jugadores de baloncesto. Universitas Psychologica, 15 (4). http://dx.doi.o rg/10.11144/Javeriana.upsy 15-4.pmpc

\section{Introducción}

La posibilidad de construir una teoría general que explique cuáles son las claves que determinan el comportamiento habilidoso en diferentes dominios constituye actualmente un gran debate científico (Ericsson, Charness, Feltovich, \& Hoffman, 2006). El estudio de la competencia experta ha sido objeto de investigación a partir de diferentes marcos de referencia. En el ámbito del deporte, la aproximación bajo el rendimiento experto constituye un enfoque de investigación sobre el que se han llevado a cabo numerosos estudios en los últimos veinte años (Hodges, Huys, \& Starkes, 2007; Starkes \& Ericsson, 2003).

Siguiendo el modelo propuesto por Williams y Ericsson (2005), el avance en el conocimiento sobre este fenómeno se desarrolla a partir de tres tipos diferentes de investigaciones sucesivas. En primer lugar, el objetivo está en capturar el rendimiento experto. Posteriormente, la intención es identificar los mecanismos subyacentes a la actuación deportiva experta. Por último, y en tercer lugar, los estudios de investigación han de ir enfocados a analizar cómo tiene lugar el desarrollo de la pericia de cada uno de los marcadores de habilidad previamente encontrados. De forma general, se ha demostrado que son cinco los elementos principales con los que se relaciona un elevado nivel de destreza en el desempeño de una actividad cognitiva: las habilidades intelectuales, la organización del conocimiento, la práctica deliberada y el contexto instruccional (Castejón, Prieto, Pérez, \& Gilar, 2004; Ericsson, 1999; Ericsson \& Charness, 1994; Ericsson, Krampe, \& Tesch-Römer, 1993; Sternberg, 1999).

Del mismo modo, los resultados de las investigaciones realizadas bajo el paradigma experto-novel, para el caso de tareas deportivas con exigencia cognitiva han puesto de manifiesto la importancia del conocimiento procedimental como una de las variables explicativas que definen la actuación exitosa en el deporte (Thomas \& Thomas, 1994). El conocimiento procedimental está identificado con el saber cómo hacer en cada momento del juego. Los sujetos con un mayor nivel de pericia disponen de un conocimiento más elaborado, estructurado, organizado y sofisticado que otros con niveles inferiores de destreza (Del Villar, Iglesias, Moreno, Fuentes, \& Cervelló, 2004; Doods, Griffin, \& Placek, 2001; Rink, French, \& Tjeerdsma, 1996). Estas estructuras superiores de conocimiento almacenadas en memoria permiten a los sujetos más hábiles tomar decisiones más rápidas y acertadas (Iglesias, Moreno, Santos-Rosa, Cervelló, \& Del Villar, 2005; Nielsen \& McPherson, 2001).

Existen evidencias empíricas sobre la importancia de la experiencia en el proceso de construcción del conocimiento procedimental (Thomas, Gallagher, \& Thomas, 2001). Pero no se conoce con precisión cómo ha de estar organizada la práctica y cuáles tienen que ser las características de la instrucción para conseguir que un sujeto disponga de una destreza cognitiva superior (Côté, Baker, \& Abernethy, 2007). También existe un déficit de investigaciones que analicen el papel de la motivación en el proceso de adquisición de la competencia (Baker \& Davids, 2007), aunque se sugiere que las experiencias prácticas tempranas contribuyen de forma importante en la motivación que los sujetos tendrán a largo plazo para mantener su continuidad en el proceso de instrucción. Este trabajo pretende profundizar en el papel de la motivación como predictor del conocimiento procedimental en el deporte. 
El papel de la motivación en la predicción del conocimiento procedimental en jugadores de balo...

En este sentido, las creencias de habilidad están estrechamente relacionadas con la motivación. La importancia del estudio de las creencias implícitas de habilidad en el ámbito físico-deportivo ha sido manifestada por diversos estudios (e. g., Biddle, Soos, \& Chatzisarantis, 1999; Chian \& Wang, 2008; Wang \& Biddle, 2001). Según estas investigaciones las creencias implícitas de habilidad que poseen las personas influyen de manera importante en el comportamiento humano. De forma concreta, las creencias de habilidad parecen determinantes del rendimiento en la adquisición de habilidades motrices (Jourden, Bandura, \& Banfield, 1991; Moreno, González-Cutre, Martín-Albo, \& Cervelló, 2010).

Las concepciones o creencias implícitas de habilidad se basan en la comprensión por parte de los sujetos de las diferencias entre capacidad y esfuerzo. Una concepción estable de habilidad representa la creencia de que la habilidad no puede ser modificada con el esfuerzo o la práctica, y la actuación depende de capacidades innatas. Mientras que una concepción modificable de habilidad implica que esta puede ser mejorada con un incremento del esfuerzo y la práctica (Nicholls, 1992). La aplicación de estos conceptos al ámbito físico-deportivo ha corrido a cargo de Biddle y sus colaboradores (Biddle et al., 1999; Biddle, Wang, Chatzisarantis, \& Spray, 2003; Sarrazin et al., 1996; Wang \& Biddle, 2001), quienes establecen dos tipos de creencias implícitas de habilidad deportiva, una creencia incremental según la cual la habilidad puede ser mejorada a través del esfuerzo y el aprendizaje, y una creencia de entidad que considera la habilidad como algo estable y, por tanto, no es susceptible de modificarse.

Según Dweck y Leggett (1988), las relaciones entre las creencias de habilidad y diferentes consecuencias cognitivas, afectivas $\mathrm{y}$ conductuales, se dan mediadas por las orientaciones de meta y moderadas por la competencia percibida. En este sentido, un deportista con una creencia incremental de habilidad tiende a desarrollar una orientación a la tarea, que se caracteriza por el esfuerzo y la búsqueda de la superación y mejora personal. Sin embargo, la creencia de entidad lleva al deportista a comparar ese nivel estable de habilidad que cree que tiene con el nivel de otros (orientación al ego). A su vez, la orientación a la tarea se relaciona positivamente con patrones adaptativos (e. g., procesamiento profundo o desarrollo del pensamiento crítico, rendimiento, búsqueda de la ayuda necesaria para completar la tarea, persistencia, motivación intrínseca, búsqueda de desafíos), mientras que la orientación al ego solo se relaciona positivamente con estos patrones adaptativos, si la percepción de competencia es alta. No obstante, algunos trabajos (González-Cutre, Sicilia, \& Moreno, 2008, Ommundsen, 2001) han mostrado que la creencia incremental también se puede relacionar con la orientación al ego. Creer que la habilidad puede mejorar no tiene por qué ser incompatible con querer demostrar mayor habilidad que los demás.

Cury, Elliot, Da Fonseca y Moller (2006), tras revisar los estudios que habían testado el modelo de Dweck y Leggett (1988), consideraron que la relación entre las creencias de habilidad y diferentes consecuencias no solo se daba mediada, sino que también se podía dar de forma directa. Cury et al. realizaron dos estudios, uno de campo y otro de laboratorio con estudiantes de entre 12 y 15 años. En el estudio de campo, se midió como consecuencia el rendimiento en matemáticas, mientras que en el estudio de laboratorio se midió el rendimiento en una prueba de inteligencia y la motivación intrínseca, tras manipular las creencias de habilidad y el feedback. La creencia incremental predijo positivamente el rendimiento en matemáticas, el rendimiento intelectual y la motivación intrínseca, mientras que la creencia de entidad lo hizo negativamente. La competencia percibida también predijo positivamente el rendimiento en matemáticas y el intelectual.

Respecto a las relaciones existentes entre las creencias de habilidad y algunas variables que podrían estar asociadas con el conocimiento procedimental, Ommundsen (2003) realizó un interesante estudio en educación física. 
Encontró que la creencia incremental predecía positivamente la elaboración de estrategias (por ejemplo, relacionar ideas), la regulación metacognitiva (abordar una situación difícil de manera diferente), la regulación del esfuerzo y la búsqueda de ayuda, mientras que la creencia de que la habilidad es estable predecía dichas variables de forma negativa.

Así, el propósito de esta investigación fue analizar el papel de la motivación en la predicción del conocimiento procedimental, estableciéndose como hipótesis que la creencia incremental, la orientación a la tarea y la percepción de competencia constituirían variables precursoras del conocimiento procedimental, mientras que las creencias de entidad y la orientación al ego no favorecerían la adquisición de un mayor conocimiento procedimental. De este modo, se podría determinar el efecto de las variables motivacionales como predictoras de los talentos en el desempeño de tareas deportivas con exigencia cognitiva.

\section{Método}

\section{Participantes}

La muestra estuvo compuesta por 339 jugadores de baloncesto (202 varones y 137 mujeres), de edades comprendidas entre los 12 y 15 años ( $M$ $=13.84, D E=1.05$ ). Del total de la muestra, el $21.7 \%$ tenía una experiencia en competición de baloncesto de un año; el $59 \%$, entre uno y tres años y el $19.3 \%$, más de tres años de experiencia. El $39 \%$ entrenaba tres horas a la semana; el 34.4 $\%$, cinco horas y el resto (26.6 \%) más de cinco horas a la semana.

\section{Instrumentos}

\section{Escala de Creencias Implícitas sobre la Habilidad (CNAAQ-2)}

Se utilizó la versión española (González-Cutre et al., 2007) del Conceptions of the Nature of Athletic Ability Questionnaire-2 (Biddle et al., 2003). Este instrumento se compone de dos factores de orden superior, Creencias incrementales y Creencias de entidad. Las Creencias implícitas incrementales se miden a través de dos subfactores: aprendizaje y mejora . El subfactor aprendizaje está compuesto por tres ítems (e. g., "Para tener éxito en el deporte se necesita aprender técnicas y habilidades, y practicarlas regularmente") y el subfactor mejora también está compuesto por tres ítems (e. g., "Como de bueno seas en deporte, podrá siempre mejorarse si trabajas para ello"). Las Creencias implícitas de entidad también se miden a través de dos factores: estable y talento . El subfactor estable está compuesto por tres ítems (e. g., "Es difícil cambiar lo bueno que tú eres en el deporte") y el subfactor talento por otros tres ítems (e. g., "Necesitas tener algún talento natural para ser bueno en deporte"). Todas las respuestas corresponden a una escala tipo Likert que varía de 1 a 5 , donde 1 corresponde a totalmente en desacuerdo y 5 a totalmente de acuerdo. La fiabilidad obtenida fue de 0.73 para la creencia de habilidad de entidad (0.68 para estable y 0.77 para talento) y 0.75 para la creencia de habilidad incremental (0.66 para mejora y 0.7 para aprendizaje). Las subescalas estable y mejora han mostrado un valor alfa inferior al recomendado (Nunnally, 1978), sin embargo, al estar compuesta por pocos ítems la validez interna observada podía ser marginalmente aceptada (Hair, Anderson, Tatham, \& Black, 1998). Los índices de ajuste obtenidos en el análisis factorial confirmatorio fueron los siguientes: $\left[\#^{2}(48, \mathrm{~N}=339)=144.34\right.$, $\mathrm{p}<0.001, \#^{2} / \mathrm{gl}=3, \mathrm{CFI}=0.91, \mathrm{NFI}=0.9, \mathrm{TLI}$ $=0.9$, SRMR $=0.06$, RMSEA $=0.07]$.

\section{Cuestionario de Percepción de Éxito (POSQ) (Roberts \& Balagué, 1991; Roberts, Treasure, \& Balagué, 1998)}

Se ha utilizado la versión en español (Cervelló, Escartí, \& Balagué, 1999; Martínez Galindo, Alonso, \& Moreno, 2006) de dicho cuestionario para medir las orientaciones de meta de los 
adolescentes en el deporte. El inventario estuvo compuesto por 12 ítems, seis de los cuales respondían al factor Orientación hacia la tarea del deportista y los otros seis, al factor Orientación hacia el ego. Las respuestas a dicho cuestionario fueron cerradas y respondían a una escala tipo Likert que oscilaba desde 1, valor que correspondía a totalmente en desacuerdo a 5 valor correspondiente a totalmente de acuerdo . Dicho cuestionario mostró valores alfa que fueron de 0.9 para la subescala ego y de 0.79 para la subescala tarea. Los índices de ajuste obtenidos en el análisis factorial confirmatorio fueron los siguientes: $\left[\#^{2}(53, \mathrm{~N}=339)=91.57\right.$, $\mathrm{p}<0.001, \#^{2} / \mathrm{gl}=1.72, \mathrm{CFI}=0.98, \mathrm{NFI}=0.95$, $\mathrm{TLI}=0.97, \mathrm{SRMR}=0.04, \mathrm{RMSEA}=0.04]$.

\section{Competencia}

Para medir la percepción de competencia se utilizó la misma estrategia seguida por Wang y Biddle (2003) consistente en la transformación de los ítems de la escala de competencia física (e. g., "Soy muy bueno jugando al baloncesto") del cuestionario que analiza el perfil de autopercepción física (Fox \& Corbin, 1989) a una escala tipo Likert que oscilaba desde 1 (totalmente en desacuerdo) a 5 ( totalmente de acuerdo ). La fiabilidad obtenida fue de 0.73.

\section{Cuestionario para la Valoración del Conocimiento Procedimental en Baloncesto (CCPB)}

Para la medición del conocimiento procedimental en baloncesto, se empleó un cuestionario de 16 preguntas de respuesta múltiple elaborado a partir de la propuesta de McGee y Farrow (1987) y validado al contexto español (Del Villar et al., 2004; Iglesias et al., 2005). En el enunciado de cada pregunta, se define una situación de juego concreta y en las respuestas aparecen diferentes posibles soluciones (e. g., “¿Qué tipo de cambio de mano durante la acción de bote emplearías para cambiar de dirección si tienes el defensor muy pegado al balón y no tienes opción de pase? a) el cambio de mano por delante; b) el reverso; c) el cambio de mano entre las piernas; d) agotaría el bote e intentaría buscar una opción de pase). La puntuación máxima que se puede obtener es 16 y la mínima 0 . Se realizó una prueba de análisis de la fiabilidad (alfa de Cronbach), obteniéndose un coeficiente de 0.74 .

\section{Procedimiento}

Se hizo contacto con el máximo responsable de las escuelas de baloncesto elegidas y los entrenadores, para informarles de los objetivos y pedirles su colaboración. Previa autorización paterna, la administración de los cuestionarios tuvo lugar estando presente el investigador principal, para hacer una breve explicación del objetivo de estudio, informar de cómo cumplimentar los instrumentos y solventar todas las dudas que pudieran surgir durante el proceso, insistiendo en el anonimato de las respuestas y en que se contestara con sinceridad y leyendo todos los ítems. El tiempo requerido para rellenar las escalas fue de aproximadamente 15 minutos, variando ligeramente según la edad del jugador.

\section{Análisis de datos}

En primer lugar, se realizaron estadísticos descriptivos y correlaciones de todas las variables. Posteriormente, se llevó a cabo un modelo de ecuaciones estructurales en dos pasos (Anderson \& Gerbing, 1988). En el primer paso, se trató de dar validez de constructo al modelo de medida, con un análisis en el que las variables correlacionaban libremente. En el segundo paso, se testó el modelo hipotetizado, que planteaba que las creencias de habilidad predecirían las orientaciones de meta y la competencia percibida y estas a su vez predecirían el conocimiento procedimental. Para comprobar el papel mediador de las orientaciones de meta y la competencia percibida, se realizó un análisis de mediación siguiendo los pasos establecidos por Baron y Kenny (1986). En este sentido primeramente, se comprobó el efecto de las 
Eduardo Cervelló Gimeno, David González-Cutre, Juan Antonio Moreno Murcia, Damián Iglesias Gallego.

variables independientes sobre los mediadores. En segundo lugar, se analizó el efecto de los mediadores sobre la variable dependiente. Por último, se trató de comprobar que controlando los efectos de las variables independientes sobre los mediadores y los efectos de los mediadores sobre la variable dependiente, disminuían los efectos de las variables independientes sobre la dependiente.

\section{Resultados}

\section{Medias, desviaciones estándar y análisis de correlación}

En la Tabla 1 se puede observar que los jugadores revelaron una mayor orientación a la tarea ( $M$ = 4.32) que al ego $(M=3.54)$ y una mayor creencia incremental de habilidad ( $M=4.22$ ) que de entidad ( $M=2.37$ ). La competencia percibida estuvo en torno al punto medio de la escala ( $M=2.92$ ), al igual que el conocimiento procedimental $(M=8.27)$.

El análisis de correlaciones mostró que la orientación a la tarea $(r=0.18, \mathrm{p}<$ $0.01)$ y la creencia incremental ( $r=0.32$, $\mathrm{p}<0.01$ ), con sus diferentes subescalas, se relacionaban positivamente con el conocimiento procedimental, mientras que la orientación al ego $(r=-0.14, p<0.01)$ y la creencia de entidad $(r=-0.28, p<0.01)$, con sus subfactores correspondientes, lo hacían negativamente. La relación entre competencia percibida y conocimiento procedimental no fue significativa.

\section{TABLA 1}

Media, desviación estándar, coeficiente alfa y correlaciones de todas las variables

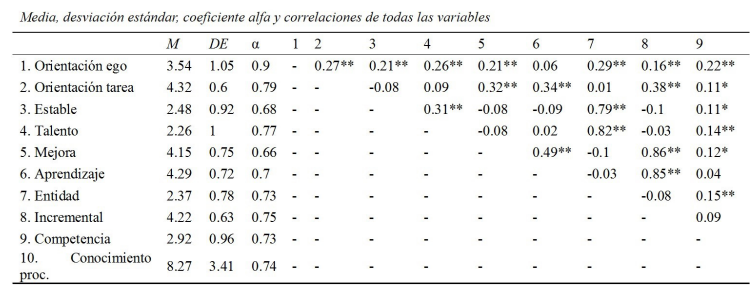

Fuente: elaboración propia

\section{Modelo de ecuaciones estructurales}

Se utilizó la matriz de covarianza como entrada para el análisis de datos. El análisis se realizó con el método de estimación de máxima verosimilitud, junto con el procedimiento de bootstrapping, puesto que el coeficiente de Mardia elevado (17.54) indicaba la ausencia de normalidad multivariada. El análisis de bootstrapping reveló que los resultados de las estimaciones eran robustos y no se veían afectados por la falta de normalidad (Byrne, 2001).

Puesto que la muestra no era muy elevada, y tratando de mantener una ratio de 1-10 entre el tamaño de la muestra y el número de parámetros del modelo (Kline, 2005), se utilizaron algunas parcelas. Para las creencias de habilidad, se utilizaron como indicadores las puntuaciones medias de sus dos subescalas (aprendizaje y mejora, para la creencia incremental, y estable y talento para entidad). Para cada una de las orientaciones de meta se parcelaron sus ítems en dos grupos homogéneos de tres, cuyas medias se utilizaron como indicadores. Para la competencia percibida se utilizaron como indicadores sus dos ítems. Puesto que la puntuación en conocimiento procedimental era exacta, su media se representó directamente como una variable observada.

Para testar el ajuste del modelo hipotetizado a la matriz de datos se utilizaron los siguientes índices de ajuste: $\#^{2}$, la ratio $\#^{2} / \mathrm{gl}$, CFI (Comparative Fit Index), NFI (Normed Fit Index), TLI (Tucker Lewis Index), SRMR (Standardized Root Mean Square Residual) y RMSEA (Root Mean Square Error of Approximation). Puesto que el $\#^{2}$ es muy sensible al tamaño de la muestra resulta conveniente tener en cuenta la ratio entre el $\#^{2}$ y los grados de libertad $\left(\#^{2} / g l\right.$.). Este valor se considera aceptable cuando es inferior a 5 (Bentler, 1989), aunque otros autores consideran que es más conveniente obtener valores entre 2 y 3 (Schermelleh-Engel, Moosbrugger, \& Müller, 2003). Los índices CFI, NFI y TLI deben ser superiores a 0.9 (Schumacker \& Lomax, 1996), aunque lo ideal son valores por encima de 0.95 
(Hu \& Bentler, 1999). Valores inferiores a 0.08 para el SRMR (Hu \& Bentler, 1999) y para el RMSEA (Browne \& Cudeck, 1993) se pueden considerar aceptables.

Los índices de ajuste obtenidos del análisis del modelo de medida fueron los siguientes: $\left[\#^{2}(30\right.$, $\mathrm{N}=339)=75.57, \mathrm{p}<0.001, \#^{2} / \mathrm{gl}=2.51, \mathrm{CFI}$ $=0.96, \mathrm{NFI}=0.94, \mathrm{TLI}=0.93, \mathrm{SRMR}=0.04$, RMSEA $=0.06]$. Las correlaciones oscilaron entre -0.43 y 0.53 . Estos resultados indicaban que el modelo de medida era aceptable.

Seguidamente, se analizaron las relaciones predictivas hipotetizadas (Figura 1). Los resultados reflejaron estos valores: $\left[\#^{2}(36, \mathrm{~N}\right.$ $=339)=139.6, \mathrm{p}<0.001, \#^{2} / \mathrm{gl}=3.87, \mathrm{CFI}$ $=0.91, \mathrm{NFI}=0.88, \mathrm{TLI}=0.86, \mathrm{SRMR}=$ 0.07$, RMSEA $=0.09]$. La creencia incremental predecía positivamente la orientación a la tarea $(\mathrm{b}=0.61)$ y la orientación al ego $(\mathrm{b}=$ 0.34 ), mientras que la creencia de entidad predecía positivamente la orientación al ego $(b=$ 0.54). Ambas creencias predecían positivamente la competencia percibida $(b=0.22$ para incremental y $\mathrm{b}=0.34$ para entidad). $\mathrm{La}$ orientación a la tarea predecía positivamente el conocimiento procedimental $(b=0.31)$, mientras que la orientación al ego lo hacía de forma negativa $(b=-0.27)$. La competencia percibida no predijo de forma significativa el conocimiento procedimental. La varianza explicada del conocimiento procedimental fue de un $14 \%$. En este modelo se pueden apreciar los efectos de las variables dependientes sobre los mediadores, y de estos sobre la variable independiente (a excepción de la competencia percibida). No obstante, los índices de ajuste no fueron muy satisfactorios.

\section{Figura 1}

Modelo de ecuaciones estructurales

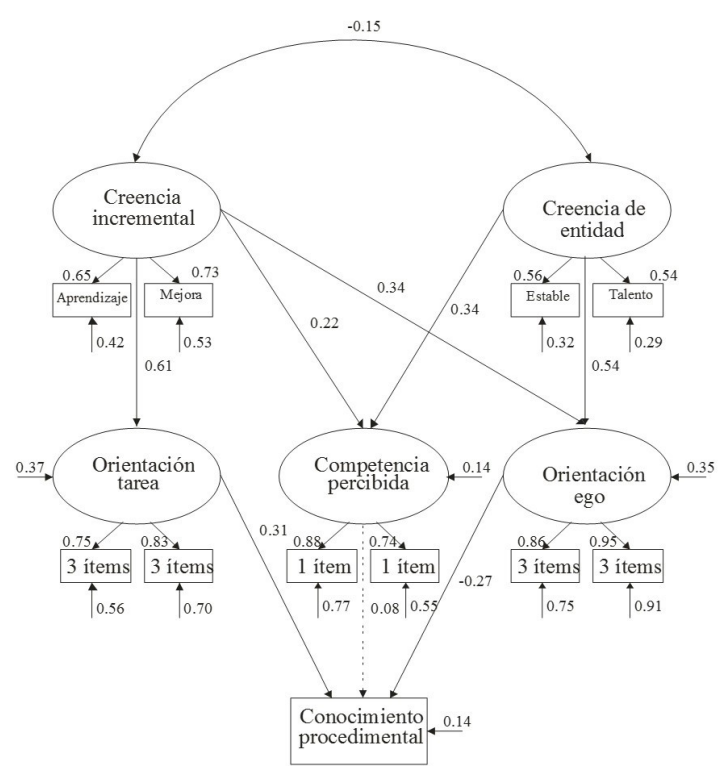

Todos los parámetros están estandarizados y son estadísticamente significativos excepto la relación entre la competencia percibida y el conocimiento procedimental, representada por una línea discontinua. Las varianzas explicadas se muestran sobre las flechas pequeñas. Fuente: elaboración propia

A continuación, para analizar los efectos de mediación, en primer lugar, testamos un modelo en el que las creencias de habilidad predecían directamente el conocimiento procedimental. Los resultados del análisis del modelo revelaron los siguientes índices de ajuste: [ $\#^{2}(3, \mathrm{~N}=339)$ $=6.2, \mathrm{p}>0.05, \#^{2} / \mathrm{gl}=2.07, \mathrm{CFI}=0.99, \mathrm{NFI}$ $=0.97, \mathrm{TLI}=0.95, \mathrm{SRMR}=0.02$, RMSEA $=0.05]$. La creencia incremental predecía positivamente el conocimiento procedimental (b $=0.35)$, mientras que la creencia de entidad lo hacía negativamente $(b=-0.36)$, con una varianza explicada del $28 \%$. Finalmente, se trató de comprobar si disminuían estos efectos de las variables independientes sobre la variable dependiente, controlando los efectos de las variables dependientes sobre los mediadores, y de estos sobre la variable dependiente (Baron \& Kenny, 1986). Para ello, se testó un modelo parcialmente mediado, en el cual las creencias predecían indirectamente el 
conocimiento procedimental a través de las orientaciones y la competencia (primer modelo hipotetizado), pero además se añadían dos parámetros que relacionaban directamente las creencias y el conocimiento procedimental. Los resultados de este modelo mostraron los siguientes índices: $\left[\#^{2}(34, \mathrm{~N}=339)=\right.$ 92.76, $\mathrm{p}<0.001, \#^{2} / \mathrm{gl}=2.72, \mathrm{CFI}=0.95$, NFI $=0.92$, TLI $=0.92$, SRMR $=0.05$, RMSEA $=0.07]$. En contra de lo esperado, los resultados revelaron que, al añadir esos parámetros directos entre las creencias y el conocimiento procedimental, la relación entre las orientaciones de meta y el conocimiento procedimental dejaba de ser significativa. La creencia incremental continuaba prediciendo positivamente el conocimiento procedimental (b $=0.37)$, mientras que la creencia de entidad lo hacía negativamente $(b=-0.39)$. La varianza explicada fue del $35 \%$. Esto indicaba que las orientaciones de meta y la competencia percibida no mediaban la relación entre las creencias y el conocimiento procedimental, sino que esta era directa.

\section{Discusión}

El objetivo de este trabajo ha sido comprobar el efecto de ciertas variables motivacionales sobre la predicción del conocimiento procedimental en el deporte. Las hipótesis de trabajo fueron que las creencias incrementales de habilidad y la orientación a la tarea predirían positivamente el conocimiento procedimental, mientras que las creencias de entidad y la orientación al ego predirían negativamente dicho conocimiento. También se esperaba una predicción positiva de la percepción de competencia sobre el conocimiento procedimental. Los resultados obtenidos han corroborado parcialmente las hipótesis de trabajo, aunque han arrojado algunos datos que son muy relevantes para el análisis de la relación entre la motivación y el conocimiento procedimental.

En primer lugar, se encontró que las creencias de habilidad son predictores más poderosos del conocimiento procedimental que las orientaciones motivacionales. De hecho, el modelo que presentaba mayor poder de predicción era aquel en el que las creencias de habilidad predecían directamente el conocimiento procedimental, y en el que las orientaciones disposicionales dejaban de presentar una predicción significativa. Este resultado es muy interesante y es necesario reflexionar sobre él. Lo que los resultados están indicando es que, independientemente de la concepción de éxito adoptada por los deportistas, lo que determina un mayor conocimiento procedimental es la creencia de que la habilidad es modificable con el entrenamiento. De manera contraria, la creencia de que la habilidad es un elemento estable, inmodificable y poco mejorable con el entrenamiento, determina un menor conocimiento procedimental. Estos resultados son similares a los obtenidos por Cury et al. (2006), en los que encontraron, al igual que en el presente trabajo, que las creencias de habilidad eran predictores directos del rendimiento en una tarea de matemáticas $\mathrm{y}$ en un test de inteligencia. Otros estudios también han mostrado que las creencias de habilidad parecen jugar un papel importante en el rendimiento en tareas motrices (Jourden et al., 1991; Moreno et al., 2010) y en el desarrollo de estrategias cognitivas (Ommundsen, 2003).

Lo que parecen estar indicando estos datos es que la forma en la que los deportistas afrontan la práctica deportiva, desde un punto de vista motivacional, puede estar modulando la forma en la que aprovechan la experiencia deportiva. Existen diversos estudios en el ámbito del conocimiento procedimental que demuestran que la experiencia es un factor determinante en la adquisición de un mayor conocimiento procedimental (Del Villar et al., 2004; French \& Thomas, 1987; McPherson, 1999a, 1999b, 2000; McPherson \& Kernodle, 2007). Sin embargo, los resultados de este estudio aportan algo más de luz sobre el efecto que la experiencia puede tener en la adquisición de este conocimiento. Parece ser que aquellas personas que viven la experiencia deportiva desde una creencia basada en que la habilidad es mejorable a lo largo del tiempo, puedan estar aplicando un "plus" a su grado de 
El papel de la motivación en la predicción del conocimiento procedimental en jugadores de balo...

experiencia, de forma que aquellos deportistas que presenten más conocimiento procedimental sean aquellos que además de la experiencia, hayan vivido esta de forma más constructiva y bajo criterios de creencias dinámicas sobre la construcción de la pericia deportiva. Este aspecto es muy relevante y debería ser considerado en la investigación futura como un elemento para tener en cuenta.

Un resultado curioso, y en cierta medida sorprendente, ha sido comprobar que la percepción de competencia no ha predicho el conocimiento procedimental, lo cual indicaría que es independiente de lo competente que se perciba el sujeto y entraría en contradicción con aquellos estudios que muestran una relación directa entre el conocimiento y el rendimiento (French \& Thomas, 1987; Iglesias et al., 2005; McPherson \& French, 1991; McPherson \& Thomas, 1989). Una cuestión que podría explicar este resultado sería la naturaleza de la medida de la percepción de competencia, un poco sesgada hacia criterios comparativos más que autorreferenciales en el contenido de los ítems. De hecho, la creencia de entidad ha predicho de forma más alta la percepción de competencia, probablemente debido al contenido de los ítems de la subescala.

Una prospectiva de futuro sería la utilización de medidas más objetivas de rendimiento, más que la percepción de competencia, con el objeto de poder determinar el efecto de la motivación sobre el conocimiento procedimental, y a su vez el efecto de este sobre el rendimiento, lo que nos daría un modelo mucho más ecológico y transferible al ámbito deportivo.

Un segundo aspecto, que no era objetivo inicial de este trabajo, ha sido comprobar el efecto directo o indirecto de las creencias de habilidad sobre el conocimiento procedimental. Existe cierta controversia en la relación que se establece entre las creencias de habilidad y las orientaciones motivacionales en la predicción de distintas consecuencias motivacionales. Cury et al. (2006) defienden que las relaciones entre las creencias y diferentes consecuencias se pueden dar tanto de forma directa como mediada a través de las metas de logro. En el estudio actual, se ha encontrado que la creencia incremental predecía positivamente la orientación a la tarea, mientras que la creencia de entidad predecía positivamente la orientación al ego.

Al igual que en otros trabajos (Gonzalez-Cutre et al., 2008; Odmmunsen, 2001), también se halló una predicción positiva desde la creencia incremental hacia la orientación al ego. Tal y como indican estos autores, resulta coincidente que este resultado exprese la posibilidad de poseer una creencia incremental de habilidad y a la vez mostrar interés por demostrar más capacidad que los demás. A su vez, la orientación a la tarea, de forma positiva, y la orientación al ego, de forma negativa, predecían el conocimiento procedimental. No obstante, el análisis de mediación posterior ha revelado que el efecto de las orientaciones de meta sobre el conocimiento procedimental se anula cuando tenemos en cuenta el efecto directo de las creencias de habilidad sobre el conocimiento procedimental. Los resultados sugieren, por tanto, que en la predicción del conocimiento procedimental las orientaciones de meta no parecen ser muy relevantes. Así pues, las creencias implícitas de habilidad predecirían directamente el conocimiento procedimental de los deportistas.

Aun así, son necesarios más estudios que traten de comprobar los resultados que se derivan de este trabajo, puesto que es uno de los primeros que ha analizado la influencia de la motivación sobre la adquisición del conocimiento procedimental. En este sentido, sería interesante analizar la mediación de las metas de logro, entre las creencias de habilidad y el conocimiento procedimental, desde una perspectiva 2 X 2 (Elliot \& McGregor, 2001). Hay que tener en cuenta que en este estudio se midieron las orientaciones de meta desde una perspectiva dicotómica (egotarea) puesto que se inició antes de que el modelo 2 X 2 fuera aplicado en España (Moreno, González-Cutre, \& Sicilia, 2008). Del mismo modo, resultaría interesante abordar el estudio del conocimiento procedimental desde el modelo jerárquico de la motivación intrínseca y extrínseca (Vallerand, 2001), analizando 
su relación con distintos factores sociales, necesidades psicológicas básicas y formas de motivación.

Resumiendo, este estudio ha supuesto una primera incursión para analizar el papel de la motivación en la predicción del conocimiento procedimental. Los resultados indican que las creencias implícitas de habilidad predicen directamente el conocimiento procedimental sin mediación de las orientaciones de meta y la competencia percibida. La creencia incremental se relaciona positivamente con el conocimiento procedimental, mientras que la creencia de entidad lo hace de forma negativa. Estos resultados señalan la importancia de hacer ver a los deportistas que su habilidad siempre puede mejorar con trabajo, entrenamiento y esfuerzo. De esta manera se podría influir de forma positiva sobre sus destrezas motrices y su rendimiento.

\section{Referencias}

Anderson, J. C., \& Gerbing, D. W. (1988). Structural equation modeling in practice: A review and recommended two-step approach. Psychological Bulletin , 103 , 411-423.

Baker, J., \& Davids, K. (2007). Sound and fury, signifying nothing? Future directions in the nature-nurture debate. International Journal of Sport Psychology, 38, 135-143.

Baron, R. M., \& Kenny, D. A. (1986). The moderator-mediator variable distinction in social psychological research: Conceptual, strategic, and statistical considerations. Journal of Personality and Social Psychology, 51, 1173-1182.

Bentler, P. M. (1989). EQS structural equations program manual . Los Angeles: BMDP Statistical Software.

Biddle, S., Soos, I., \& Chatzisarantis, N. (1999). Predicting physical activity intentions using a goal perspectives approach: A study of Hungarian youth. Scandinavian Journal of Medicine and Science in Sports , 9 , 353-357.
Biddle, S. J. H., Wang, C. K. J., Chatzisarantis, N. L. D., \& Spray, C. M. (2003). Motivation for physical activity in young people: Entity and incremental beliefs about athletic ability. Journal of Sports Sciences , 21 (12), 973-989.

Browne, M. W., \& Cudeck, R. (1993). Alternative ways of assessing model fit. En K. A. Bollen \& J. S. Long (Eds.), Testing structural equation models (pp. 136-162). Newbury Park, CA: Sage.

Byrne, B. M. (2001). Structural equation modeling with Amos: Basic concepts, applications, and programming . Mahwah, NJ: Erlbaum.

Castejón, J. L., Prieto, M. D., Pérez, A. M., \& Gilar, R. (2004). El rol del conocimiento $\mathrm{y}$ de las habilidades intelectuales generales en la adquisición del aprendizaje complejo. Psicothema , 16 (4), 600-605.

Cervelló, E. M., Escartí, A., \& Balagué, G. (1999). Relaciones entre la orientación de metas disposicional y la satisfacción con los resultados deportivos, las creencias sobre las causas de éxito en deporte y la diversión con la práctica deportiva. Revista de Psicología del Deporte , 8, 7-19.

Chian, L. K. Z., \& Wang, C. K. J. (2008). Motivational profiles of junior college athletes: A cluster analysis. Journal of Applied Sport Psychology , 20 (2), 137-156.

Côté, J., Baker, J., \& Abernethy, B. (2003). Practice and play in the development of sport expertise. En J. L. Starkes \& K. A. Ericsson (Eds.), Expert performance in sports: Advances in research on sport expertise (pp. 184-202). Champaign, IL: Human Kinetics.

Cury, F., Elliot, A. J., Da Fonseca, D., \& Moller, A. C. (2006). The social-cognitive model of achievement motivation and the $2 \times 2$ achievement goal framework. Journal of Personality and Social Psychology, 90 (4), 666-679.

Del Villar, F., Iglesias, D., Moreno, M. P., Fuentes, J. P., \& Cervelló, E. M. (2004). An investigation into procedural 
knowledge and decisión-making: Spanish experienced-inexperienced basketball players differences. Journal of Human Movement Studies , 46 , 407-420.

Dodds, P., Griffin, L. L., \& Placek, J. H. (2001). A selected review of the literature on development of learners' domainspecific knowledge. Journal of Teaching in Physical Education, 20 (4), 301-313.

Dweck, C. S., \& Leggett, E. L. (1988). A social-cognitive approach to motivation and personality. Psychological Review , 95 , 256-273.

Elliot, A. J., \& McGregor, H. A. (2001). A 2 X 2 achievement goal framework. Journal of Personality and Social Psychology, 80 (3), 501-519.

Ericsson, K. A. (1999). Creative expertise as superior reproductible performance: Innovative and flexible aspects of expert performance. Psychological Inquiry, 10 , 329-361.

Ericsson, K. A., \& Charness, N. (1994). Expert performance: Its structure and acquisition. American Psychologist , 49 (8), 725-747.

Ericsson, K. A., Charness, N., Feltovich, P. J., \& Hoffman, R. R. (Eds.). (2006). The Cambridge handbook of expertise and expert performance. Nueva York: Cambridge University Press.

Ericsson, K. A., Krampe, R. T., \& Tesch-Römer, C. (1993). The role of deliberate practice in the acquisition of expert performance. Psychological Review, 100, 363-406.

Fox, K. R., \& Corbin, C. D. (1989). The Physical Self-Perception Profile: Development and preliminary validation. Journal of Sport and Exercise Psychology, 11 , 408-430.

French, K. E. \& Thomas, J. R. (1987). The relation of knowledge development to children's basketball performance. Journal of Sport Psychology, 9 , 15-32.

González-Cutre, D., Martínez Galindo, C., Alonso, N., Cervelló, E., Conte, L., \& Moreno, J. A. (2007). Las creencias implícitas de habilidad y los mediadores psicológicos como variables predictoras de la motivación autodeterminada en deportistas adolescentes. En J. Castellano \& O. Usabiaga (Eds.), Investigación en la actividad física y el deporte II (pp. 407-417). Vitoria: Universidad del País Vasco.

González-Cutre, D., Sicilia, A., \& Moreno, J. A. (2008). Modelo cognitivo-social de la motivación de logro en educación física. Psicothema, 20 (4), 642-651.

Hair, J. F., Anderson, R. E., Tatham, R. L., \& Black, W. C. (1998). Multivariate data analysis . Upper Saddle River, NJ: Prentice-Hall.

Hodges, N. J., Huys, R., \& Starkes, J. L. (2003). Methodological review and evaluation of research in expert performance in sport. En J. L. Starkes \& K. A. Ericsson (Eds.), Expert performance in sports: Advances in research on sport expertise (pp. 161-183). Champaign, IL: Human Kinetics.

Hu, L., \& Bentler, P. M. (1999). Cutoff criteria for fit indexes in covariance structure analysis: Conventional criteria versus new alternatives. Structural Equation Modeling , 6, 1-55.

Iglesias, D., Moreno, M. P., Santos-Rosa, F. J., Cervelló, E. M., \& Del Villar, F. (2005). Cognitive expertise in sport: Relationships between procedural knowledge, experience, and performance in youth basketball. Journal of Human Movement Studies, 49, 65-76.

Jourden, F. J., Bandura, A., \& Banfield, J. T. (1991). The impact of conceptions of ability on self-regulatory factors and motor skill acquisition. Journal of Sport and Exercise Psychology, 13 , 213-226.

Kline, R. B. (2005). Principles and practice of structural equation modeling (2.a ed.). Nueva York: Guildford Press.

Martínez Galindo, C., Alonso, N., \& Moreno, J. A. (2006). Análisis factorial confirmatorio del "Cuestionario de Percepción de Éxito (POSQ)" en alumnos adolescentes de Educación Física. En M. A. González, J. A. Sánchez \& A. Areces (Eds.), IV Congreso de la Asociación Española de Ciencias del 
Eduardo Cervelló Gimeno, David González-Cutre, Juan Antonio Moreno Murcia, Damián Iglesias Gallego.

Deporte (pp. 757-761). A Coruña: Xunta de Galicia.

McGee, R., \& Farrow, A. (1987). Test questions for physical education activities . Champaign, IL: Human Kinetics.

McPherson, S. L. (1999a). Expert-novice differences in performance skills and problem representations of youth and adults during tennis competition. Research Quarterly for Exercise and Sport, 70 , 233-251.

McPherson, S. L. (1999b). Tactical differences in problem representations and solutions in collegiate varsity and beginner women tennis players. Research Quarterly for Exercise and Sport, 70 , 369-384.

McPherson, S. L. (2000). Expert-novice differences in planning strategies during collegiate singles tennis competition. Journal of Sport and Exercise Psychology , $22,39-62$.

McPherson, S. L., \& French, K. E. (1991). Changes in cognitive strategy and motor skill in tennis. Journal of Sport and Exercise Science, 13, 26-41.

McPherson, S. L., \& Kernodle, M. (2007). Mapping two new points on the tennis expertise continuum: Tactical skills of adult advanced beginners and entry-level professionals during competition. Journal of Sports Sciences, 25 (8), 945-959.

McPherson, S. L., \& Thomas, J. R. (1989). Relation of knowledge and performance in boys' tennis: Age and expertise. Journal of Experimental Child Psychology, 48 , 190-211.

Moreno, J. A., González-Cutre, D., \& Sicilia, A. (2008). Metas de logro 2 X 2 en estudiantes españoles de Educación Física. Revista de Educación, 347 , 299-317.

Moreno, J. A., González-Cutre, D., MartínAlbo, J., \& Cervelló, E. (2010). Motivation and performance in physical education: An experimental test. Journal of Sports Science and Medicine, 9 , 79-85.

Nicholls, J. G. (1992). The general and the specific in the development and expression of achievement motivation. En G. C.
Roberts (Ed.), Motivation in sport and exercise (pp. 31-56). Champaign, IL: Human Kinetics.

Nielsen, T. M., \& McPherson, S. L. (2001). Response selection and execution skills of professionals and novices during singles tennis competition. Perceptual and Motor Skills, 93 , 541-555.

Nunnally, J. C. (1978). Psychometric theory . Nueva York: McGraw-Hill.

Ommundsen, Y. (2001). Self-handicapping strategies in physical education classes: The influence of implicit theories of the nature of ability and achievement goal orientations. Psychology of Sport and Exercise, 2 , 139-156.

Ommundsen, Y. (2003). Implicit theories of ability and self-regulation strategies in physical education classes. Educational Psychology, 23 , 141-157.

Rink, J. E., French, K. E., \& Tjeerdsma, B. L. (1996). Foundations for the learning and instruction of sport and games. Journal of Teaching in Physical Education, 15 , 399-417.

Roberts, G. C., \& Balagué, G. (septiembre, 1991). The development and validation of the Perception of Success Questionnaire. Paper presented at the FEPSAC Congress, Cologne, Germany.

Roberts, G. C., Treasure, D. C., \& Balagué, G. (1998). Achievement goals in sport: The development and validation of the Perception of Success Questionnaire. Journal of Sports Sciences, 16, 337-347.

Sarrazin, P., Biddle, S. J. H., Famose, J. P., Cury, F., Fox, K., \& Durand, M. (1996). Goal orientations and conceptions of the nature of sport ability in children: A social cognitive approach. British Journal of Social Psychology, 35 , 399-414.

Schermelleh-Engel, K., Moosbrugger, H., \& Müller, H. (2003). Evaluating the fit of structural equation models: Tests of significance and descriptive goodness-offit measures. Methods of Psychological Research Online, 8 (2), 23-74. 
Schumacker, R. E., \& Lomax, R. G. (1996). A beginner's guide to structural equation modeling . Mahwah, NJ: Erlbaum.

Starkes, J. L., \& Ericsson, K. A. (Eds.). (2003). Expert performance in sports: Advances in research on sport expertise. Champaign, IL: Human Kinetics.

Sternberg, R. J. (1999). Intelligence as developing expertise. Contemporary Educational Researcher, 24 , 359-375.

Thomas, K. T., Gallagher, J. D., \& Thomas, J. R. (2001). Motor development and skill acquisition during childhood and adolescence. En R. N. Singer, H. Hausenblas \& C. M. Janelle (Eds.), Handbook of sport psychology (2. ${ }^{a}$ ed., pp. 20-52). Nueva York: John Wiley \& Sons.

Thomas, K. T., \& Thomas, J. R. (1994). Developing expertise in sport: The relation of knowledge and performance. International Journal of Sport Psychology , $25,295-315$.

Vallerand, R. J. (2001). A hierarchical model of intrinsic and extrinsic motivation in sport and exercise. En G. C. Roberts (Ed.), Advances in motivation in sport and exercise (pp. 263-319). Champaign, IL: Human Kinetics.

Wang, C. K. J., \& Biddle, S. J. H. (2001). Young people's motivational profiles in physical activity: A cluster analysis. Journal of Sport and Exercise Psychology, 23 , 1-22.

Wang, C. K. J., \& Biddle, S. J. H. (2003). Intrinsic motivation towards sports in Singaporean students: The role of sport ability beliefs. Journal of Health Psychology, 8, 515-523.

Williams, A. M., \& Ericsson, K. A. (2005). Perceptual-cognitive expertise in sport: Some considerations when applying the expert performance approach. Human Movement Science, 24 , 283-307.

\section{Notas}

* Artículo de investigación. 